\title{
Experimental Investigation of Extraction of Active Ingredients of Scrophularia striata Boiss (as a Medicinal Herb) via Supercritical Fluid Extraction Method with Ultrasonic Waves
}

\author{
Nabilvaeli ,Bizhan honarvar*, Nadia esfandiari, Zahra arab \\ Nabilvaeli@miau.ac.ir \\ Honarvar@miau.ac.ir \\ Esfandiari_n@miau.ac.ir \\ Aboosadi@miau.ac.ir
}

Department of Chemical Engineering, marvdasht branch, Islamic Azad University, marvdasht, Iran

\begin{abstract}
One of the major problems faced by modern societies are various infectious diseases with different roots, most of which are supposed to be treated using chemical antibiotics. Considering side effects of these drugs and the fact that those fail to realize any positive influence in many cases, an ever increasing deal of attention has been paid to the utilization of medicinal herbs which exhibit great therapeutic properties without any side effect. This important characteristic of the medicinal herbs doubles the significance of the method used to extract active ingredients from the herbs in such a way to end up with optimal efficiency. In the present research, extraction of extract from Scrophularia striata Boiss, as a medicinal herb which is rich in active ingredients with antibacterial and anti-depression effects (isorhamnetin3-o-rutinoside, quercetine, cinnamic acid, nepitrin), using supercritical $\mathrm{CO}_{2}\left(\mathrm{SC}-\mathrm{CO}_{2}\right)$ with the help of ultrasonic waves, and also applying the same method without the use of the ultrasonic waves were investigated. Due to its low volatility, the medicinal extract in this plant is difficult to collect and extract. In the present research, it was observed that the yield of active ingredient extraction using the presented method was about $68 \%$ higher when ultrasonication was further applied, as compared to the case where no ultrasonic wave was applied. Thermodynamic conditions used in the present research included the pressure values of 150, 180, and 210 bar, and temperatures of 45,55 , and $65^{\circ} \mathrm{C}$, with the ultrasonication device operated at frequencies of 37 and $80 \mathrm{kHz}$ and power of 50 and $100 \mathrm{~W}$. The results indicated that, when the pressure and temperature were set to 180 bar and $65^{\circ} \mathrm{C}$, respectively, while USCF system was operated at $37 \mathrm{kHz}$, an output efficiency of $87 \%$ (in mass) was obtained, while the efficiency was as low as $41 \%$ under the same set of conditions but without ultrasonication.
\end{abstract}

Keywords: extraction, ultrasound, supercritical fluid, Scrophularia striata Boiss plant. 


\section{Introduction}

Scientifically known as Scrophularia striata, (Teshnedari Plant is of the family Scrophulariaceae which grows in western parts of Iran. Not all chemical ingredients of the plant are identified, but for years, local residents of Ilam Province (Iran) has been using this plant in various forms (e.g. edible tea, smoke, and plaster) for treating different diseases. Drinking Teshnedari tea has been used as a treatment frustration and digestive disorders and pains. Washing with cooled tea or applying its smoke have exhibited significant effects on the treatment of eye inflammation and infection pains, hemorrhoids, episiotomy, skin burns, and ear inflammation and infection pains. Preparing a mixture of the plant, animal fat, and natural gum and placing that on wound, or washing the wound with cooled tea or smoking the wound with the plant can heal infectious wounds. By preparing a mixture of the Teshnedari plant, ash tree leaf, and flour to make a plaster and applying that on the desired position, one can heal boils (as a disease). This plant contains active anti-bacterial and ant-inflammatory ingredients whose proper identification and extraction are of paramount importance. Among conventional extraction methods, traditional ones such as distillation using Clevenger or soxhlet. Although these methods have apparently high efficiency, as compared to other methods, yet the most significant problem with these methods is that these are water-based which necessitates subsequent heating (which degrades the temperature-sensitive active ingredients) and concentration by chemicals (remnants of which lower the purity of the active ingredients). Therefore, it is needed to move toward a method which not only eliminates the above-mentioned problems, but also provides a significant extraction efficiency. One of the most applicable methods for extracting sensitive medicinal compounds from plants is the supercritical fluid extraction (SFE) method.

A supercritical fluid is the one at a pressure and temperature above its critical pressure and temperature, respectively. In terms of transfer properties, these fluids behave like gases (e.g. high diffusivity and low viscosity), and in terms of solubility power, they resemble liquid solvents. In the region above the critical point wherein the fluid is said to be in supercritical conditions, there is no distinction between gas and liquid phases and density of liquid phase becomes equal to that of gaseous phase. Given that the pressure is higher than critical pressure in supercritical fluid (SCF) extraction processes, in contrary to the operations performed in liquid phase, variable pressure takes part in process control. It should be noted that, close to the critical point, small changes in pressure and temperature can end up with large variations in density. Due to very suitable characteristics of these fluids for a wide spectrum of matters, these have been applied for purification, extraction and separation purposes. Carbon dioxide and water are the most common and well-known SCFs in use [Sodeifian, Honarvar et al., 2017].

By changing temperature and pressure, one can drive a fluid toward more liquid or gaseous characteristics. The most important property of these fluids is their solubility which is directly related to their density at constant temperature, and increases with increasing the density. Given that the density is directly related to pressure, one can stipulate that, solubility of these fluids increases with increasing pressure. The relationship between solubility and temperature is somewhat more complicated. At constant density, solubility increases with increasing temperature, while small changes in temperature close to the critical point results in great fluctuations in density. Therefore, close to the critical point, with increasing temperature, solubility of these fluids usually begins with a decrease but increases subsequently [Honarvar et al., 2013].

Such a fluid is said to be neither a gas nor a liquid, but is rather better to be introduced as an intermediate phase. The phase has a solving power comparable to fluids while its common transfer properties are those of gases. The SCF used in the present research is $\mathrm{CO}_{2}$. It was selected considering its low price, chemical stability, neutrality, inflammability, stability under irradiative applications, nontoxicity, and environmental acceptability (as it can be easily returned to the system).

Low viscosity, high diffusivity, and very high buoyancy of a SCF which results in a very high density at the interface translate into better transfer properties of this fluid compared to conventional solvents. Solubility behavior of the fluid is closer to that of liquid phase, while diffusion into solid is provided by the gas-resembling transfer characteristics of the fluid. In this condition, extraction and phase separation rates can be enhanced significantly, as compared to conventional extraction processes. The important point to note is that, surface tension of a SCF is zero, facilitating the access to fine pores on 
a solid material. One of the major advantages of SCF extraction is that, solubility of solute in a solvent changes with temperature and pressure [Honarvar et al., 2013].

One of the problems faced when using a SCF as a solvent for extraction is the usually slow kinetic process. Solubility of many matters in a SCF is lower than that in the fluids commonly used in conventional extraction processes. This will lower the mass transfer rate. The traditional approach followed to accelerate the separation and extraction process has been the use of mechanical agitation systems and tools. Another approach in the respect is to utilize high-intensity ultrasonication process.

High-intensity ultrasounds are elastic waves with frequencies above $20 \mathrm{kHz}$ and intensities above 2 $\mathrm{W} / \mathrm{cm}$. Ultrasonic radiation represent a suitable solution for creating deep agitation and enhancing mass transfer processes, because the equipment used in some of the mechanisms adopted in SFE process (compression micro-flow, depressurization, heating, and cavitation) $[4,3]$ may not simply allow for using mechanical stirrers. As such, ultrasonic waves are very good alternatives in this respect. Investigation of influences of high-intensity ultrasound in kinetic and supercritical extraction are among the objectives of this process.

During the past couple of years, ultrasonic technology has been identified as a useful tool for food processing applications (d'Alessandro et al., 2012) and the respective field of research has been highly active. Two widely different approaches have been studied in relation to exploitation of ultrasound; the approaches have been related mainly to frequency and vibration intensity of the ultrasound. One of these approaches refers to low-frequency ultrasonication and has its focus particularly on product and process quality control (e.g. evaluation of fermentation time for cheese or mixed analysis (Da Porto C et al., 2013). Applications of this approach are mainly related to ultrasound velocity measurement

Signal attenuation, or frequency spectrum analysis. In these applications, there is no impact on the process or product. Another approach, i.e. low-frequency high-power ultrasonication, has been successfully employed to improve a process or product quality. This has been implemented for creating processes (Ghitescu R et al., 2015), maceration microbial and enzymatic inactivation (Honarvar B et al., 2013), freezing processes (Zhang 2009), content equalization (Corrales, 2008), extraction of natural products (Vikho et al., food cutting, emulsion quality enhancement (Volf I et al., 2008), slurry preparation (sugar substitution processes and fermentation processes etc.

Of the advantages of using ultrasonication (which was originally investigated by Misson et al. in 1996) is its potential application for extracting medicinal herbs and oils (carnosic acid, active ingredients of ginseng, carvone, limonene, anthraquinone, amaranth oil, gingerol, soya, almond oil, apricot oil), proteins (soybean protein), and well-known bioactive ingredients. These compounds are composed of plants and herbs (polyphenols, anthocyanins, tartaric acid, perfume compounds, polysaccharides, and applied compounds) or animal ingredients (chitin and lutein) [42]. Recently, the design of ultrasonication equipment has been advanced toward presenting powerful industrial process ability.

Presented advantages include the followings:

1. A general increase in extraction efficiency or yield,

2. Increased extraction of aqueous processes wherein no solvent can be used (water concentrate processing),

3. Providing an opportunity for using GRAS as an alternative to solvents for improving their extraction performance,

4. Accessibility of inexpensive resources/alternative resources of raw material while preserving the bio-activity level, and

5. Enhanced recovery of heat-sensitive parts, which otherwise end up with low or inacceptable efficiency.

Enhanced extraction with the help of ultrasonic waves results in the ultrasonic pressure waves and thereby cavity forces, where bubbles collapse explosively and create local pressures which result in the tearing of the plant texture, improving intra-cellular contents diffusion into the solvent. 
According to collision between high-speed particles, and disordering the micro-diffusible particles of biomass. In addition, cavitation in the vicinity of the solid-liquid contact transmits a fast-moving flow of liquid to the surface, while cavitation on the device surface results in collision with fine particles obtained from surface exfoliation, erosion, and particle decomposition.

In the present research, active ingredients of Scrophularia striata (Figure 1) were extracted using supercritical $\mathrm{CO}_{2}$ extraction at three pressures $(150,180$, and 210 bar), three temperatures $(45,55$, and $65^{\circ} \mathrm{C}$ ), and different particle sizes (mesh sizes pf 40,60, and 80) with and without USCF ultrasonic waves. The two cases were compared to one another and effects of thermodynamic conditions and the power of the ultrasonic waves on these two methods were evaluated in different cases.

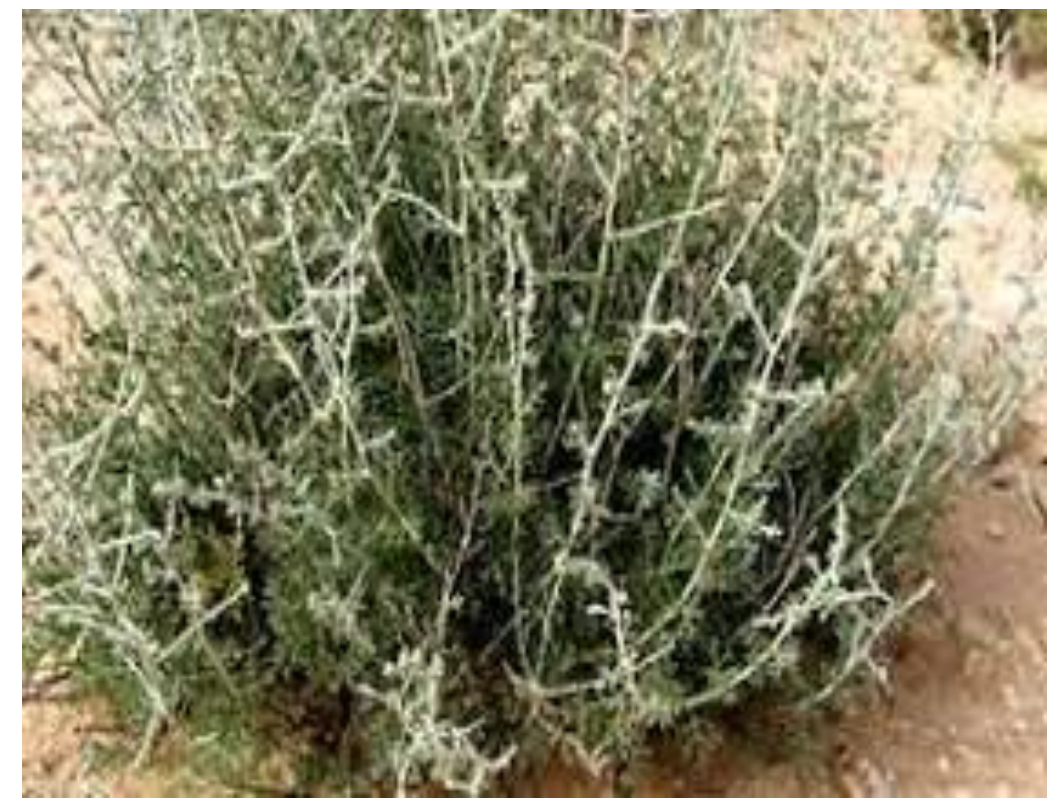

Figure 1. An image of Scrophularia striata plant.

\section{Materials and methods}

\subsection{Materials}

Scrophularia striata plant (Teshnedari) was collected from the area around the city of Ilam (Iran). Once finished with collecting the plant, about $200 \mathrm{~g}$ of that was well dried. Its stems were separated and milled to three mesh sizes of 40,60, and $80 \mu \mathrm{m}$. Furthermore, $\mathrm{CO}_{2}$ at $99 \%$ purity was used as SCF in this research.

\subsection{Supercritical fluid extraction}

A multistage system was used to perform SFE (Figure 2). The first stage involved the tank containing $\mathrm{CO}_{2}$ gas which entered the refrigerator upon opening the valve (wherein its temperature was lowered to $-15^{\circ} \mathrm{C}$ ). This was done to liquefy the $\mathrm{CO}_{2}$ gas, so as to be able to provide the desired pressure in the pumping stage. Following condensation, the gas was stored in a gas tank. The next stage was composed of a special piston pump which increased the liquefied $\mathrm{CO}_{2}$ gas pressure to a predetermined value. There was a valve at outlet of the pump which was used to control the amount of gas introduced into the extraction column cell. The extraction column was a two-walled part made from stainless steel (height $=23 \mathrm{~cm}$, inner diameter $=2.8 \mathrm{~cm}$, and outer diameter $=5.3 \mathrm{~cm}$ ). In order to keep warm the outer wall, hot water (heated by a preinstalled heater) was pumped around the column. In order to investigate the effect of ultrasonic waves, the extraction vessel was placed inside an ultrasonic bath (ELMA, Germany) 
operated at $310 \mathrm{~W}$, and extraction was performed at frequencies of 37 and $80 \mathrm{kHz}$ and powers of 50 and $100 \mathrm{~W}$.

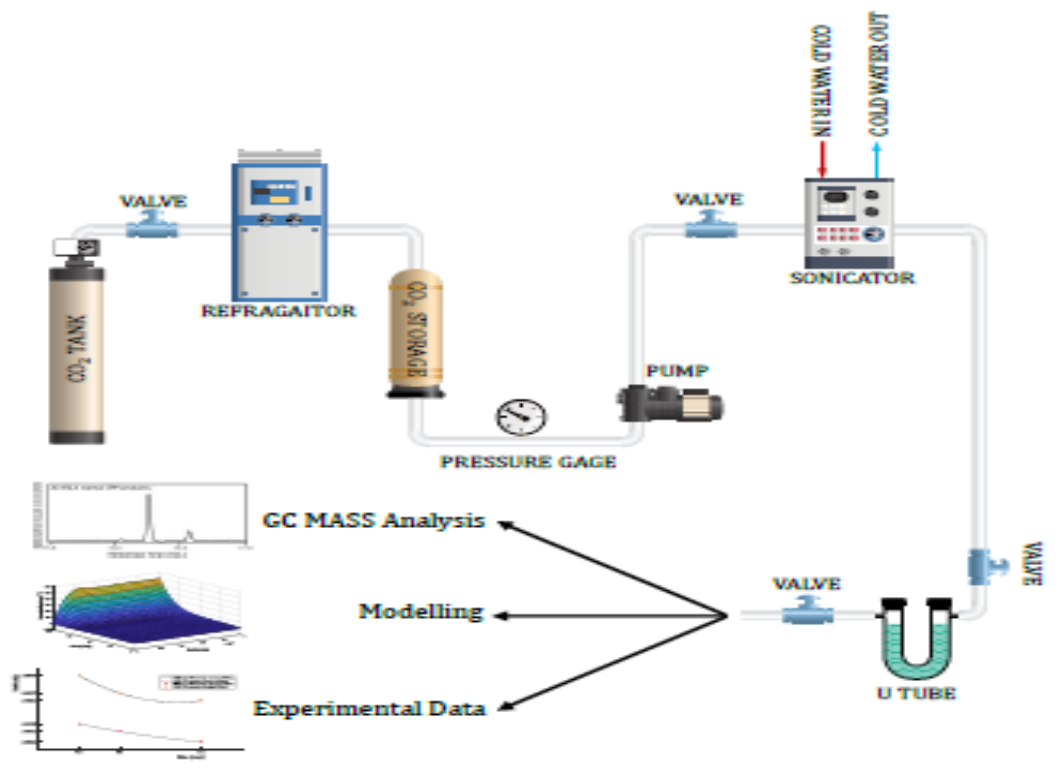

Figure 2. SFE apparatus diagram.

\subsection{Methodology}

Firstly, dried and milled plant was sieved using mesh numbers 40,60 , and 80 to obtain $5 \mathrm{~g}$ of each sample for experimental stage. In order to determine adequate number of experiments, the experimental work was designed by Taguchi's method in Minitab Software (Table 1). At each stage, after placing the sample in the extraction column cell, $\mathrm{CO}_{2}$ gas storage valve was opened, so that the gas could enter the refrigerator for about $40 \mathrm{~min}$. Gas retention time in this chamber depends on the $\mathrm{CO}_{2}$ storage pressure. Following the condensation, the gas entered liquefied gas storage and then to the reciprocating pump to have its pressure raised to supercritical pressure. By opening the outlet valve, the fluid entered the extraction column cell which was equipped with two walls. As warm water (provided by the heaterequipped vessel) entered into inner shell of the extraction cell, the fluid reached the predetermined supercritical temperature. The static extraction time was set to $40 \mathrm{~min}$. Once the product outlet valve was opened, some dichloromethane was loaded in the storage vessel to collect the volatile product of the process. The storage vessel was further stored in an ice bath to keep the product from escaping. 
Table 1. Design of experiment via Taguchi's method

\begin{tabular}{ccccc}
\hline Pressure(bar) & Temperature $\left({ }^{0} \mathrm{C}\right)$ & Particle Size $(\mathrm{mm})$ & Frequency $(\mathrm{Hz})$ & Ey\% \\
\hline 150 & 45 & 0.1 & 0 & 2.5912 \\
150 & 55 & 0.2 & 37 & 2.5871 \\
150 & 65 & 0.4 & 80 & 2.6452 \\
180 & 45 & 0.2 & 80 & 2.8641 \\
180 & 55 & 0.4 & 0 & 2.7423 \\
180 & 65 & 0.1 & 37 & 3.8963 \\
210 & 45 & 0.4 & 37 & 2.9714 \\
210 & 55 & 0.1 & 80 & 3.1324 \\
210 & 65 & 0.2 & 0 & 3.2335
\end{tabular}

\subsection{Specifications of gas chromatography - mass spectroscopy (GC/MS) apparatus}

In the present research, an Aglient 5975C GC/MS apparatus equipped with a mass probe with electron ionization vessel coupled with an Aglient $7890 \mathrm{GC}$ apparatus equipped with a HP-5MS column of $30 \mathrm{~m}$ in length, $0.25 \mathrm{~mm}$ in inner diameter, and $0.25 \mu \mathrm{m}$ in film thickness was used. Inlet temperature of the $\mathrm{GC}$ apparatus was set to $28^{\circ} \mathrm{C}$, mass probe ionization vessel temperature was set to $150^{\circ} \mathrm{C}$, analyzer (quadruple) temperature was adjusted at $230^{\circ} \mathrm{C}$, and temperature of the interface within the GC/MS was set to $280^{\circ} \mathrm{C}$. Volatile compounds were measured using an experimental procedure called head-space method. Extraction yields of active ingredients under different sets of thermodynamic conditions are demonstrated in Figures 3 and 4.

\subsubsection{Volatile compounds determination using head-space method}

One of the biggest problems with volatile materials is to trap and collect them. As such, in order to maximize the percentage of volatile contents scanned by GC/MS apparatus, the experimentally extracted materials were stored in a special vial called head-space vial. The vial was then heated (at 120 $-150^{\circ} \mathrm{C}$ ) to have all of volatile contents of the extract collected on top of the vial. Afterwards, the sample required for the GC/MS apparatus was collected from top of the vial using a special syringe and then injected into the apparatus.

\section{Discussion and conclusion}

In the present research, thermodynamic parameters (pressure and temperature) and their influences on yield of extraction via SFE were investigated. For this purpose, pressures in the range of $150-210$ bar were selected, because pressure is one of the most effective parameters in enhancing extraction efficiency in SFE, so that an increase in pressure can enhance the efficiency significantly. Furthermore, lower pressures were also considered to investigate the effect of ultrasonic waves on efficiency in this range of pressure as well and compare that to those at higher pressures. As a thermodynamic variable, temperature variations impose direct effects on density, thereby reducing (or leaving unchanged) the value of efficiency. Moreover the effect of particle size on extraction efficiency was considered to determine optimal particle size. Further, in order to determine the effect of ultrasonic waves on extraction yield, influences of variable frequency and operating power of the ultrasonication device were also studied.

\subsection{Effect of pressure}

In studying the effect of pressure, experimental conditions were similar to the above-mentioned cases, except that, the extraction pressure was considered as variable. Temperature of SFE and USFE processes was set to 45,55 , and $65^{\circ} \mathrm{C}$ at different stages. As can be seen on Figure 3, the extraction efficiency increased evidently when the extraction pressure was increasing in range of $180-210$ bar. The increase in pressure increased density of the solvent while reducing the pressure of the solute vapor. This was further evident for the case where no ultrasonic waves was applied. But in the case where ultrasonication was utilized, an increase in pressure beyond 18 bar imposed no significant effect on 
efficiency. This shows that, solubility of the solute is largely dependent on the density of the supercritical fluid which exhibits very similar behavior to that of pressure in the temperature range of $45-55^{\circ} \mathrm{C}$. Therefore, any further increase in pressure results in no significant effect on the extraction efficiency. In this study, the preferred pressure for USFE process was found to be $180 \mathrm{bar}$, at which pressure the extraction efficiency was found to be about $87 \%$. In contrast, the preferred pressure for SFE process was found to be $210 \mathrm{bar}$, at which pressure the extraction efficiency was determined to be about 63\%. Results of Figure 5 show that, in the SFE process, the presence of ultrasonic waves can enhance mass dispersion, and also demonstrate that, the effect of ultrasonication is stronger at lower pressures. This results can also be interpreted as follows: compared to SFE process, USFE process can achieve the same efficiency at lower values of pressure.( Figure4)

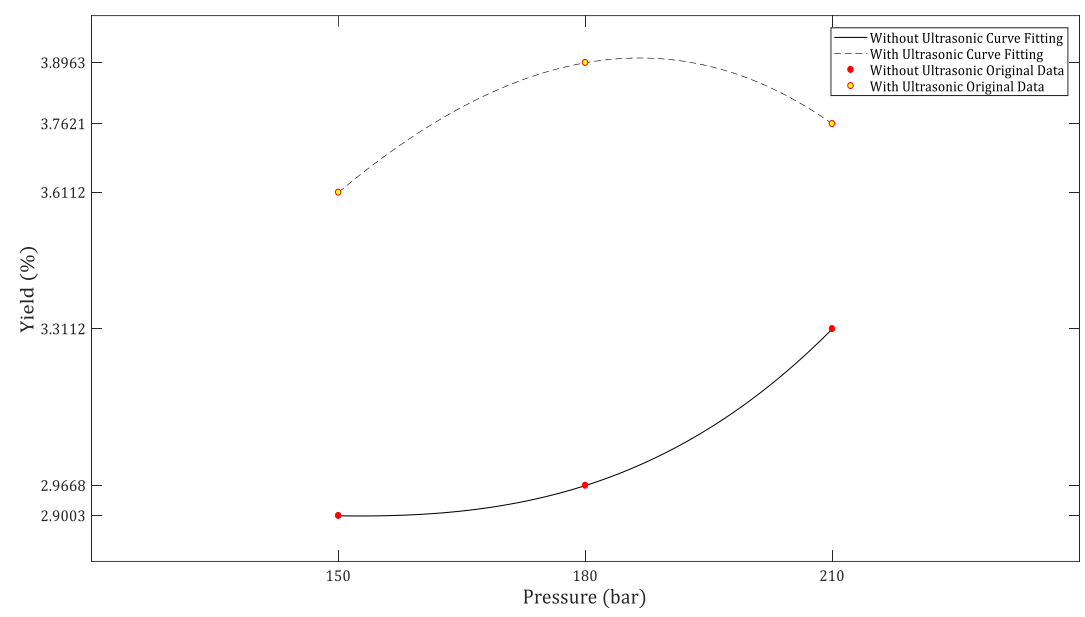

Figure (3): Effect of extraction pressure on the yield

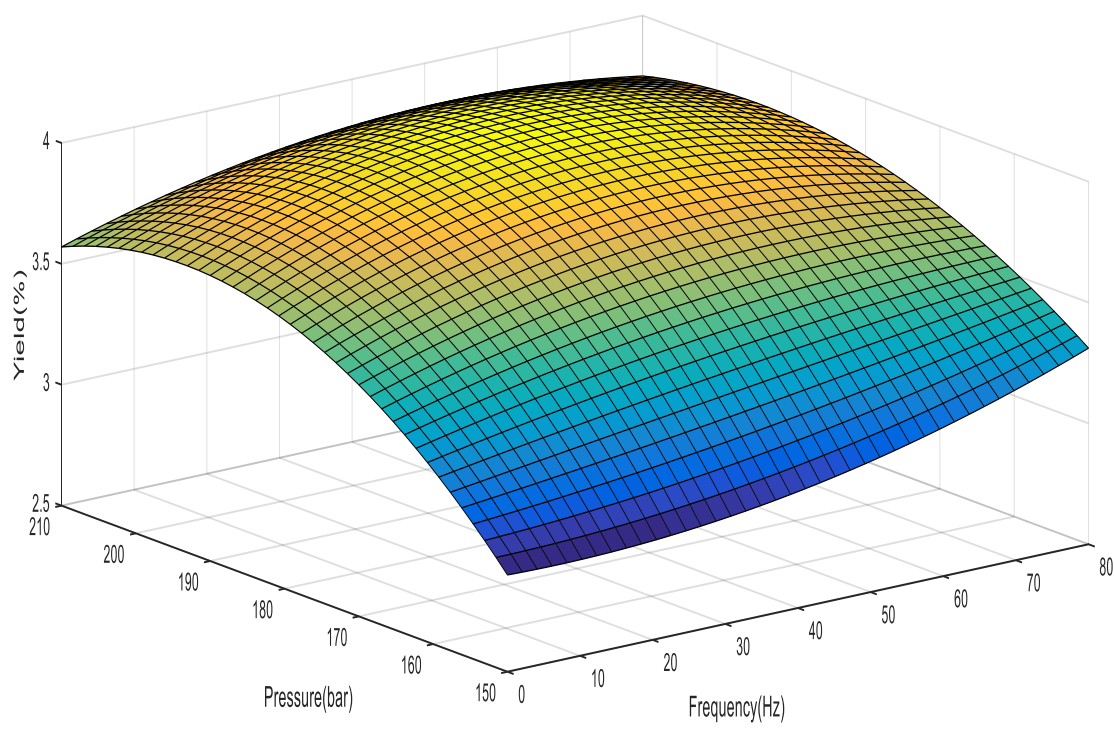

Figure (4): 3D Plot of yield V.S frecuency(HZ) V.S pressure(bar) 


\subsection{Effect of temperature}

Given that temperature affects physical and chemical properties of products and that influences of ultrasonic waves are in relation with the product properties, temperature seems to be a key variable. This is particularly true when some of the considered compounds are sensitive to temperature or volatile.

Extraction temperature affects the extraction process in two aspects: diffusivity and solubility of the solute. When temperature increases, solubility of the solute increases, thereby improving EY. However, the effect of temperature variations on the solute solubility is complicated. When temperature increases, density of $\mathrm{CO}_{2}$ decreases, thereby decreasing its solubility. On the other hand, the solute vapor pressure increases with temperature, resulting in enhanced solubility of the solute. In general, any solute-solvent system exhibits a particular apparent behavior against temperature. Optimal points for USFE and SFE processes reflect combine effects of temperature on solubility and diffusivity of the solute. The operations performed at either higher or lower temperatures than the optimal temperature will end up with lower EY values.

On the other hand, ultrasonication can improve general mass dispersion across a supercritical system. Therefore, an increase in extraction temperature improves mass transfer. In the meantime, increased temperature may be translated into lower production of cavitation energy conversion due to surface tension and increased vapor pressure (Coigpres et al., 2002). However, usually, the increase observed in the amount of extraction products can be related to increased diffusivity of solute in cells and enhanced desorption and solubility. It is also commonly observed that, with increasing the temperature, the amount of extracted product decreases (particularly in unstable and volatile compounds. In the present research, as can be seen from Figure 4, as far as SCF process is concerned, an increase in temperature to $55^{\circ} \mathrm{C}$ increased the obtained efficiency, with the yield following a decreasing trend at higher temperatures. In the graph for USCF process, the increasing trend of efficiency with temperature is evident. Setting higher temperatures in ultrasonic technique may increase the rate of bubble collapse, thereby enhancing the extraction efficiency.(figure6)

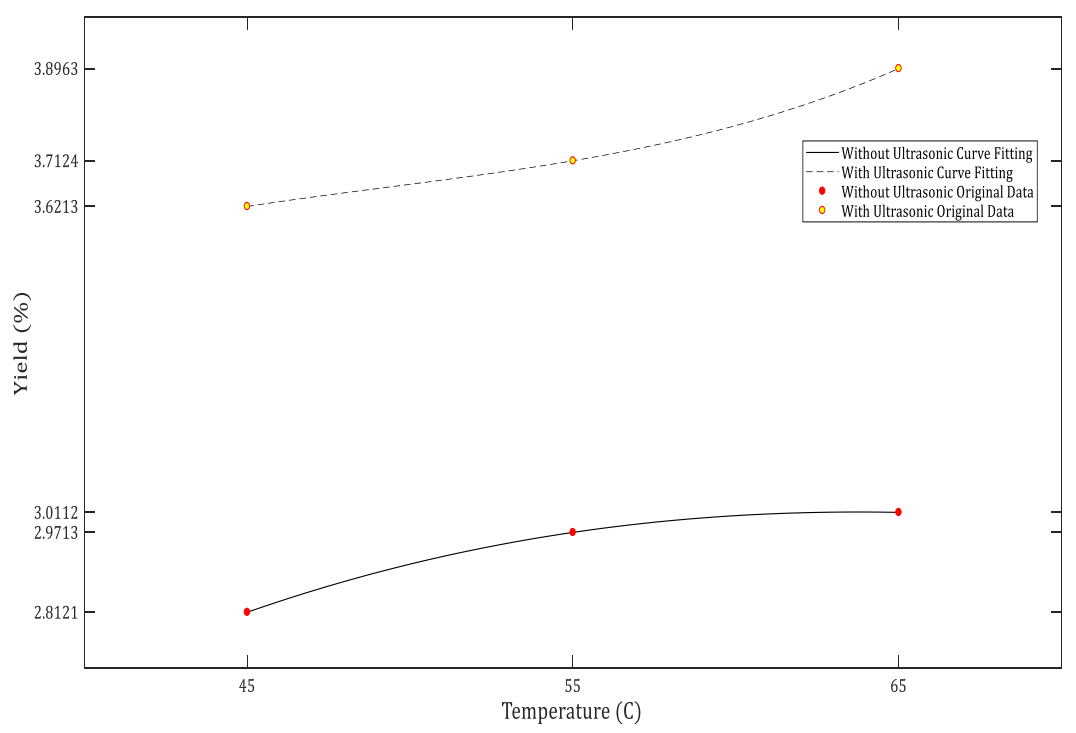

Figure (5): Effect of extraction temperature on the yield 


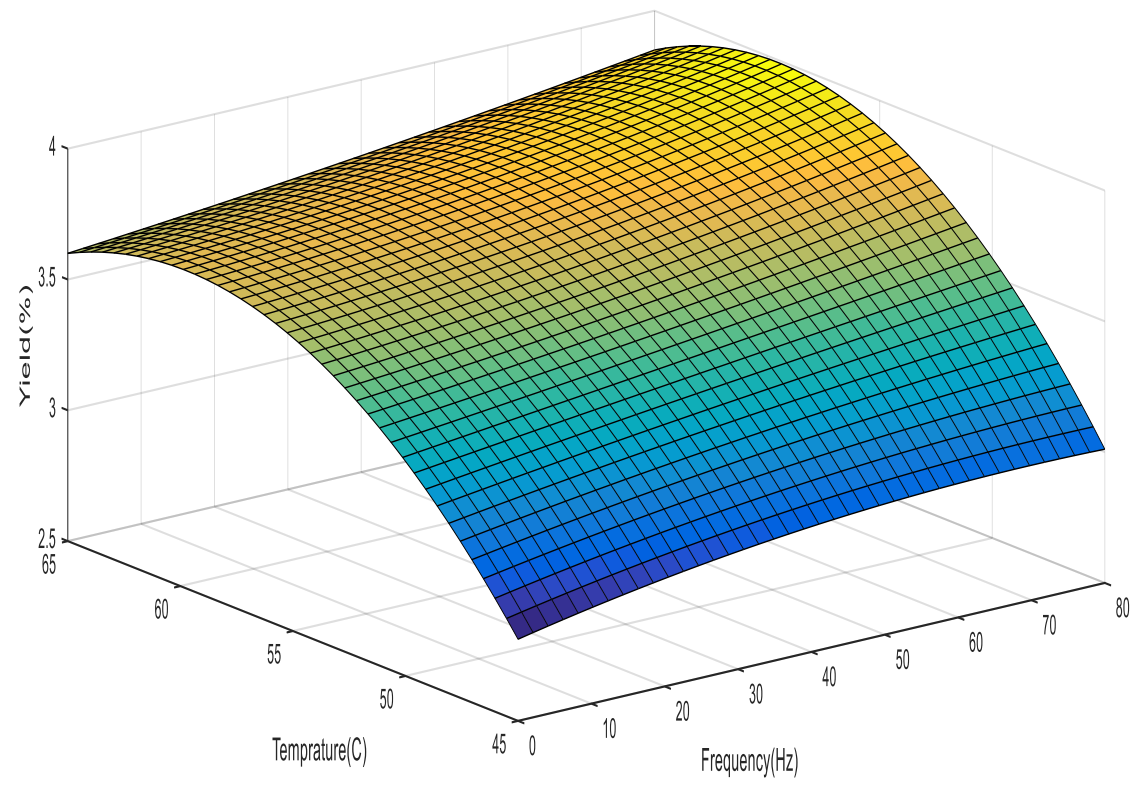

Figure (6): 3D Plot of yield V.S frecuency(HZ) V.S temperature(C)

\subsection{Effect of particle size}

The amount of extracted matter can be further influenced by particle size, because the extraction is performed in relation to the predetermined level and the pass through which the molecules shall move. For instance, in oil extraction from tobacco seeds (frequency $=40 \mathrm{kHz}$, extraction temperature $=25^{\circ} \mathrm{C}$, solvents $=$ hexane or oil ether, extraction time $=60 \mathrm{~min}$, volumetric ratio of raw material-tosolvent $=31 \mathrm{~g} / \mathrm{mL}$, applied power $=150 \mathrm{~W}$, in an ultrasonic cleaner), it was observed that, maximum yield of extraction was obtained with particles of $0.4-0.5 \mathrm{~mm}$ in size (Stanisavoljik et al., 2007). The oil obtained from the largest particles $(>0.5 \mathrm{~mm})$ was lower than that from smaller particles in the same time interval. Authors concluded that, larger amounts of oil can be transferred to total extracted liquid from larger seeds rather than smaller ones. This is due to further resistance to oil transfer in relation to longer paths. Other authors (Xao et al., 2007) notice the same behavior in their own systems and argued that, when particles are adequately small, more cell walls are likely to be broken by ultrasonic waves, so that diffusion may not be a significant stage in the extraction of these particles. Excessive decrease in particle size may not be translated into further increase in the extraction yield .The amount of the extracted material can also be affected by the particle size since the extraction is associated with the surface and the length of the path along which the molecules should move. Bubble formation at particle surface enhances combination and this increases the surface and consequently the extraction of more active ingredients. Bubbles are formed by theblockage of air molecules in the particle pores through the strike of ultrasound waves passing on these fluid particles of the bubbles. The larger the bubbles are, the greater the combination wave induced by their explosion will be. Hence, the smaller the particle size 
is, the more the pores are and the greater the number of confined molecules will be, even though, the particle morphology also has a direct impact on this issue.

This finding is also evident in the present research (Figure 7-8).

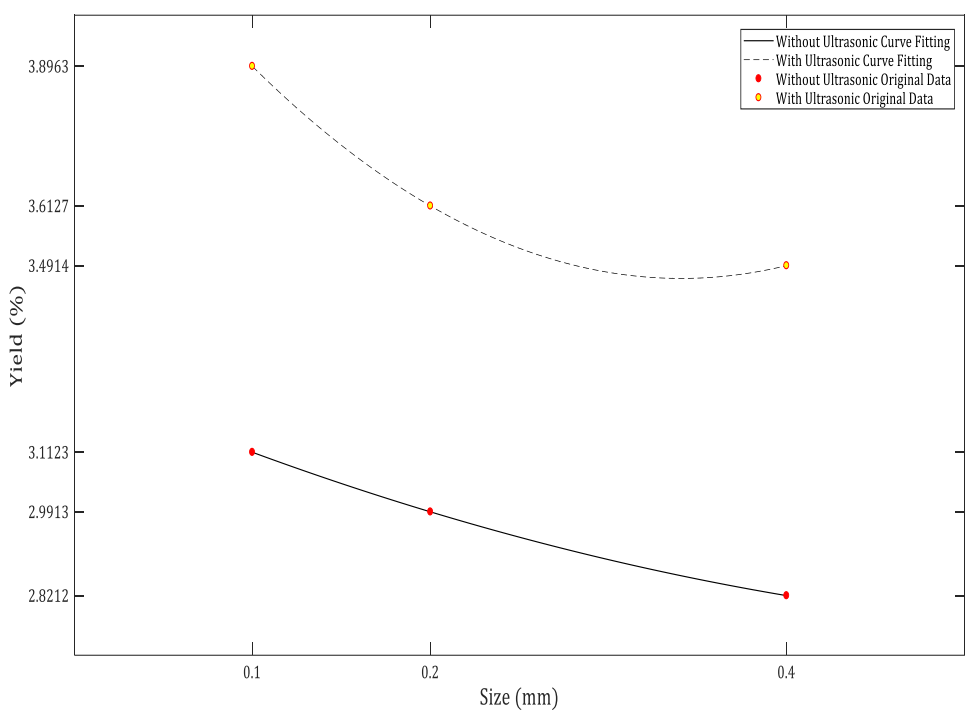

Figure (7): Effect of extraction particle size on the yield

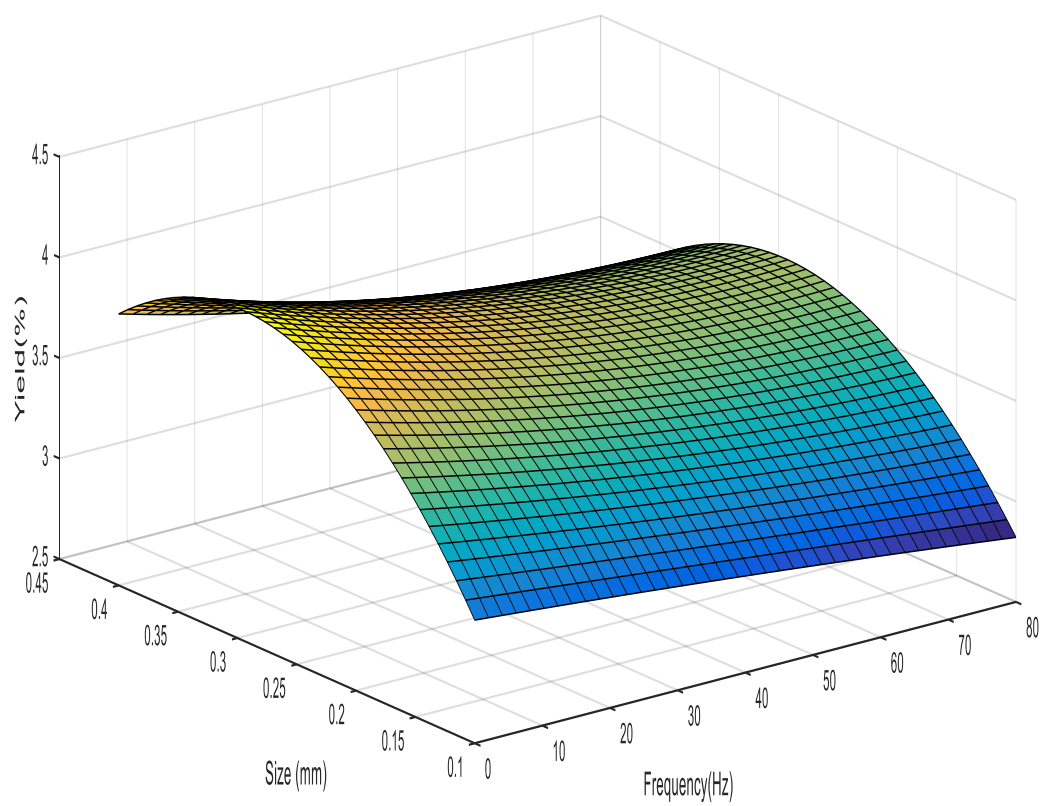

Figure (8): 3D Plot of yield V.S frecuency(HZ) V.S size(m) 


\subsection{Effect of ultrasonication frequency and power}

Increasing the extraction efficiency of organic compounds by ultrasonic methods is attributed to the formation of bubbles as a result of passing ultrasound waves through the solvent. During the application of ultrasound technique, bubbles are formed and compressed. Increasing pressure and temperature leads to bubble bursting and decomposition. Bubble bursting triggers the formation of a strike wave in the solvent and thus increases the combination speed. Ultrasonic also imposes a mechanical effect and allows the solvent to penetrate more into the sample tissue, increasing the contact area between the solid and liquid phases. This is accompanied by increased mass transfer and significant cellular degradation. Bubble explosion make the compound be released from the cell to the solvent. The waves are much more unruffled at a frequency of $37 \mathrm{kHz}$ than a frequency of $80 \mathrm{kHz}$, resulting in the formation of larger bubbles. At the frequency of $80 \mathrm{kHz}$, however, the bubbles are not allowed to be fully formed due to the speed of the strikes and vibrations and will burst before they grow up, resulting in less combination compared to the frequency of $37 \mathrm{kHz}$ (Figure9).

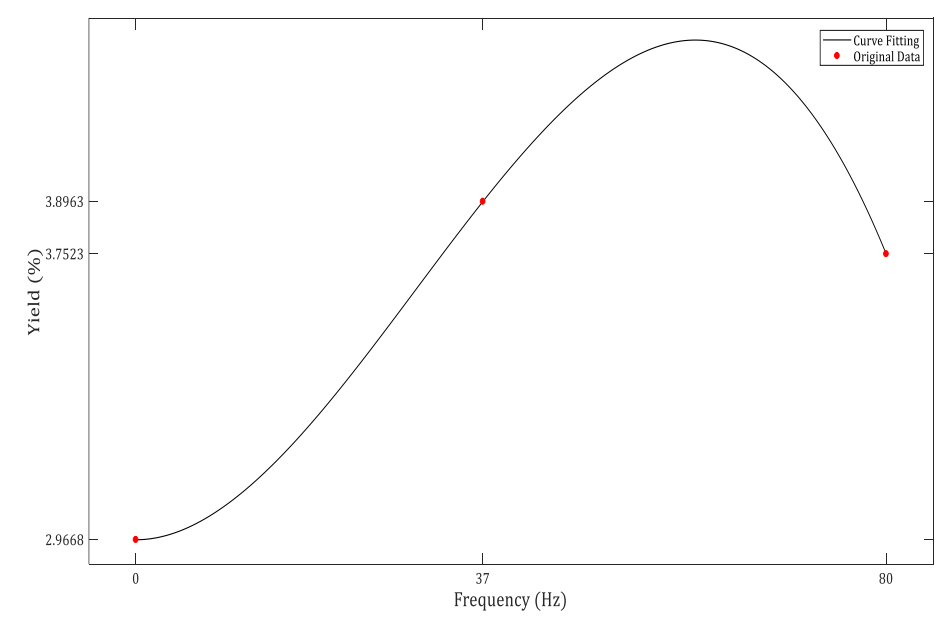

Figure (9): Effect of extraction frequency on the yield

\subsubsection{Ultrasonic wave frequency}

This is obvious that, the lower the frequency, the larger will be the cavitation bubbles. Therefore, lower frequency of powerful ultrasonic waves (about $20 \mathrm{kHz}$ ) intensify the bubble collapse, making them more efficient for extraction processes. As long as the extraction system has a complicated heterogeneous matrix of high porosity, the effect of bubble size on extraction efficiency shall be considered. The influence of frequency may be related to not only the cavitation bubble size, but also its effect on internal and external resistance to mass transfer. Although this subject matter has not been considered in extraction-related studies, but similar works have been done in the scope of drying. In that process, solid matrix plays the primary role in the effects attained by exploiting the ultrasonic wave. Figure 8 shows efficiency at frequencies of 37 and $80 \mathrm{kHz}$ and ultrasonication powers of 50 and $100 \mathrm{~W}$ 


\section{Conclusion}

Thanks to easy installation and low maintenance costs, causing return on investment within a short period, using the technique of extraction through ultrasound waves is considered as a method for extraction of valuable natural compounds. This technique requires less energy and lower solvent use, and is easier to work with. In this research, the extraction efficiency of the active ingredient was investigated at pressures of 180, 150, and 210 bars, and the results demonstrated that use of the ultrasonic process in supercritical extraction is more efficient even at low pressure than use of the nonultrasonic process at high pressure, where the increase is about 18 percent. Furthermore, temperature increase at the temperature modes used in the research by 65,55 , and 45 degrees centigrade where the uscf process was used caused an increase in efficiency by about 24 percent. The best thermodynamic conditions reported in the research included a pressure of 180 bars, a temperature of 65 degrees centigrade, a particle size of 40 micrometers, and a frequency of 37 kilohertz. Therefore, it will be effective from both aspects of energy cost reduction and significant application efficiency increase to use the ultrasound process in supercritical extraction.

\section{References}

Sodeifian, G. and Sajadian, S.A. and Honarvar, B, Mathematical modelling for extraction of oil from Dracocephalum kotschyi seeds in supercritical carbon dioxide. Natural Product Research, 1-9 (2017).

Honarvar, B. and Sajadian, S.A. and Khorram, M. and Samimi, A., Mathematical modeling of Supercritical fluid extraction of oil from canola and sesame seeds. Brazilian Journal of Chemical Engineering, $159-166$ (2013).

Vilkhu, K. and, Mawson, R. and Simins, L. and Bates, D., Applications and opportunities for ultrasound assisted extraction in the food industry - a review. Innovative FoodSci. Emerg Technol, 9, 161-169 (2008).

Corrales, M. and Toepf, S. and Butz, P. and Knorr, D. and Tauscher, Extraction of anthocyanin's from grape by-products assisted by ultrasonic, high hydrostatic pressure or pulsed electric fields: a comparison. Innovative FoodSci. Emerg. Technol, 9, 85-91 (2008).

Zhang, Q.A. and Zhang, Z.Q. and Yue, X.F. and Fan, X.H. and Li, T. and Chen, S.F., Response surface optimization of ultrasound-assisted oil extraction from autoclaved almond powder. Food Chem, 116 (2), 513-518 (2009).

Mason, J. and Paniwynyk, L. and Lorimer, P., The use of ultrasound in food technology, Sonochem. Ultrasound ,3, 253-260 (1996).

d'Alessandro, L.G. and Kriaa, K. and Nikov, I. and Dimitrov, K., Ultrasound assisted extraction of polyphenols from black chokeberry, Separation and Purificatio Technology, 93, 42 - 47 (2012).

Da Porto, C. and Porretto, E. and Decorti D., Comparison of ultrasound-assisted extraction with conventional extraction methods of oil and polyphenols from grape (Vitis vinifer $a$ L.) seeds, Ultrasonic Sonochemistry, 20, 1076- 1080 (2013).

Ghitescu, R. E. and Volf, I. and Carausu, C. and Bühlmann ,A. M. and Gilca, I.A. and Popa ,V.I., Optimization of ultrasound-assisted extraction of polyphenols from spruce wood bark, Ultrasonic Sonochemistry, 22, 535-41 (2015). 
International Academic Conference on Applied Research in Engineering, Science \& Technology

$14^{\text {th }}-15^{\text {th }}$ September 2018 Brussels, Belgium

Honarvar, B. and Sajadian, S. A. and Khorram, M. and Samimi, A., Mathematical modeling of Supercritical fluid extraction of oil from canola and sesame seeds. Brazilian Journal of Chemical Engineering, 159 - 166 (2013).

Popa, V.I. and Dumitru, M. and Volf, I. and Anghel, N., Ligninand polyphenols as allelochemicals, Industrial Crops and Products, 27, 144-149 (2008). 